\title{
Comportement dynamique des paliers-butées de ligne d'arbres soumis à des défauts géométriques
}

\author{
Olivier Bonneau ${ }^{1, a}$, SÉbastien Berger ${ }^{2}$ et Jean Frêne ${ }^{1}$ \\ 1 Laboratoire de Mécanique des Solides, UMR 6610, SP2MI, Boulevard Marie et Pierre Curie, Téléport 2, BP 30179, \\ 86962 Futuroscope-Chasseneuil Cedex, France \\ 2 Laboratoire M.I.P.S., 12 rue des Frères Lumière, 68093 Mulhouse Cedex, France
}

Reçu le 18 mai 2002, accepté le 23 octobre 2003

\begin{abstract}
Résumé - Cet article fait la synthèse de différents travaux mettant en évidence l'influence que peut avoir un défaut géométrique (de forme ou de position) d'un élément support de ligne d'arbre sur le comportement dynamique du rotor. Sous certaines conditions aussi bien dans le cas des paliers que dans les cas des butées axiales, un défaut géométrique de la partie tournante du support peut faire apparaître des perturbations non négligeables de l'ensemble de la ligne d'arbre. Après une présentation des différents modèles adoptés, quelques études de cas sont exposées. Il s'agit d'un calcul non linéaire (pas à pas dans le temps) de la ligne d'arbre flexible. Le modèle prend en compte simultanément le comportement non linéaire hydrodynamique du support fluide et le comportement flexible de l'arbre. Les comportements mis en évidence font apparaître dans les réponses de l'arbre des fréquences supersynchrones dues aux défauts des supports. Ces réponses peuvent avoir des amplitudes du même ordre de grandeur que celles obtenues sous l'effet d'un balourd.
\end{abstract}

Mots clés : Paliers hydrodynamiques / butées hydrodynamiques / défauts géométriques / arbre flexible / analyse non linéaire

\begin{abstract}
Dynamical behavior of bearings and thrust bearings submitted to geometrical defects. The goal of this article is to make a synthesis of several works done on the influence of a geometrical defects of bearings on the dynamical behaviour of flexible shaft. It appears that in some cases a geometrical defect creates important dynamical perturbations. Several models are presented and then results obtained with them will be exposed. It is non linear calculus of flexible shaft. The model take into account simultaneously the non linear behaviour of bearings and the flexibility of the shaft. A modal technique is used to reduce the number of degrees of freedom. Results show that bearing defects introduce supplementary perturbation. The linear approach is unable to show that.
\end{abstract}

Key words: Journal bearing / thrust bearing / defects / flexible shaft / modal technique / non linear analysis

\section{Introduction}

L'analyse du comportement dynamique des lignes d'arbre montées sur support fluide donne lieu au développement de deux grandes familles d'étude :

- des études orientées vers la dynamique de ligne d'arbre [1,2] pour lesquelles l'effort est mis sur la description de la structure. Cette modélisation par éléments finis permet alors d'effectuer une analyse modale de l'ensemble. Les paliers et supports fluides de ligne d'arbre sont introduits de façon simplifiée. Leur comportement est modélisé sous la forme de raideurs

\footnotetext{
a Auteur correspondant : bonneau@lms.univ-poitiers.fr
}

et d'amortissements (linéarisation de leur comportement au voisinage d'une position d'équilibre) ;

- la deuxième famille d'étude concerne une approche détaillée du comportement des éléments fluides. Un certain nombre de phénomènes peut être introduit (effet thermique, effet de turbulence du fluide...). Le comportement dynamique de palier peut en particulier être étudié par une approche non linéaire qui s'avère indispensable pour certaines applications. Il s'agit en général de simuler le comportement d'un arbre simplifié (une masse ou un solide en mouvement) dans un palier fonctionnant dans un régime non linéaire (grands déplacements dus à des charges importantes ou dus à des instabilités des positions d'équilibre) [3-5]. 
Nomenclature

\begin{tabular}{|lll|}
\hline $\mathrm{C}$ & le jeu radial & $\mathrm{m}$ \\
$(\mathrm{e}, \psi)$ & position polaire de l'arbre par rapport au stator & $\mathrm{m}, \mathrm{rd}$ \\
$\mathrm{P}$ & pression dans le film fluide & $\mathrm{Pa}$ \\
$\mathrm{H}=h+\delta_{r a}$ & épaisseur du film dans le palier avec défaut & $\mathrm{m}$ \\
$\mathrm{h}=\mathrm{C}+\mathrm{e} \cos (\theta)$ & épaisseur du film dans le palier sans défaut & $\mathrm{m}$ \\
$\mathrm{R}_{\mathrm{a}}$ & rayon de l'arbre & $\mathrm{m}$ \\
$\mathrm{R}_{\mathrm{c}}$ & rayon du coussinet & $\mathrm{m}$ \\
$(x, y)$ & position cartésienne de l'arbre/stator & $\mathrm{m}, \mathrm{m}$ \\
$\phi, \dot{\phi}, \ddot{\phi}$ & angle, vitesse, accélération angulaire du rotor & $\mathrm{rd}, \mathrm{rd} \cdot \mathrm{s}^{-1}, \mathrm{rd} \cdot \mathrm{s}^{-2}$ \\
$\delta_{\mathrm{ra}}$ & défaut radial sur l'arbre & $\mathrm{m}$ \\
$\mu$ & viscosité du fluide lubrifiant & $\mathrm{Pa} \cdot \mathrm{s}^{-1}$ \\
$\theta$ & paramètre angulaire avec origine sur la ligne des centres & $\mathrm{rd}$ \\
$\omega_{\mathrm{a}}$ & vitesse angulaire de l'arbre & $\mathrm{rd} \cdot \mathrm{s}^{-1}$ \\
$\left\{F_{p}\right\}$ & vecteur force de pesanteur & $\mathrm{N}$ \\
$\left\{F_{p e r}\right\}$ & vecteur force des perturbations & $\mathrm{N}$ \\
$\left\{F_{N L}\right\}$ & vecteur des forces non linéaires (force film => arbre) & $\mathrm{N}$ \\
$\{\delta\},\{\dot{\delta}\},\{\ddot{\delta}\}$ & vecteurs déplacement, vit., accél. des nœuds du rotor & $\mathrm{m}, \mathrm{m}^{-1} \mathrm{~s}^{-1}, \mathrm{~m} \cdot \mathrm{s}^{-2}$ \\
{$\left[C_{C}\right]$} & matrice de Coriolis, antisymétrique & $\mathrm{N} \cdot \mathrm{s}^{2} \cdot \mathrm{m}^{-1}$ \\
{$\left[K_{r}\right]$} & matrice raideur de l'arbre, symétrique & $\mathrm{N} \cdot \mathrm{m}^{-1}$ \\
{$\left[K_{c}\right]$} & matrice raideur de Coriolis, antisymétrique & $\mathrm{N} \cdot \mathrm{s}^{2} \cdot \mathrm{m}^{-1}$ \\
{$[M]$} & matrice masse du rotor, symétrique & $\mathrm{kg}$ \\
$\left(\mathrm{U}_{2}, \mathrm{~V}_{2}\right)$ & vitesse d'un point courant de l'arbre & $\mathrm{m} \cdot \mathrm{s}^{-1}$ \\
\hline
\end{tabular}

Ces deux approches sont très intéressantes, elles permettent d'une part de simplifier les problèmes et d'autre part d'isoler les phénomènes. Cependant il est maintenant indispensable de les rapprocher de façon à prendre en compte les couplages éventuels. Ces couplages permettent d'expliquer des phénomènes qui seraient sinon incompréhensibles.

Un modèle de comportement dynamique non linéaire de lignes d'arbres flexibles a été développé [6-8]. Ce modèle a tout d'abord été validé dans le cas d'un rotor flexible (type moteur d'avion) monté sur film amortisseur (squeeze film damper). Une comparaison théorie expérimentation a été effectuée sur cette application fortement non linéaire et la validation a été très bonne. Ce travail a permis d'expliquer certains phénomènes qui semblaient aller à l'encontre des résultats obtenus avec les modèles classiques.

Différents phénomènes de couplage fluide-structure ont alors été étudiés : paliers à jeu variable [9], palier alimenté par fluide électrorhéologique [10]. Nous nous proposons ici de mettre en évidence l'influence d'un défaut de forme du palier sur le comportement de la ligne d'arbre. Deux types de supports fluides vont être présentés : analyse d'un palier avec défaut et d'une butée axiale avec défaut de forme.

Le modèle de calcul de la ligne d'arbre va être décrit puis les résultats issus de deux applications seront présentés.

\section{Modélisation de la ligne d'arbre}

Ce paragraphe présente le modèle utilisé pour décrire le couplage fluide structure. Le détail du calcul des éléments fluides sera donné lors de la présentation des deux applications. La démarche générale est la suivante : la connaissance de la géométrie du film d'huile et de la cinématique associée permet de déterminer le champ de pression s'appliquant sur toute la surface de l'élément et d'obtenir l'action mécanique exercée par le fluide sur l'arbre. Ce champ de pression est obtenu par résolution (différences finies) de l'équation de Reynolds.

Le rotor est discrétisé par des éléments finis de type rotor à quatre degrés de liberté par nœud.

Soit $\{\delta\}$ le vecteur des déplacements nodaux du rotor. Le système différentiel s'écrit :

$$
\begin{aligned}
{[M]\{\ddot{\delta}\}+\dot{\phi}\left[C_{C}\right]\{\dot{\delta}\}+\left(\left[K_{r}\right]+\ddot{\phi}\left[K_{c}\right]\right)\{\delta\}=} \\
\left\{F_{p}\right\}+\left\{F_{p e r}\right\}+\left\{F_{N L}\right\}
\end{aligned}
$$

$\left\{F_{N L}\right\}$ représente les actions non linéaires dues au film fluide des supports.

Il est clair que le système obtenu présente $4(n+1)$ degrés de liberté. Une résolution itérative pas à pas demande donc un temps de calcul important. Ce temps de calcul peut être fortement diminué à l'aide d'une réduction modale du système.

Il s'agit de travailler dans une base ne comportant que les premiers modes de vibration de la structure [1] (les 6 premiers modes sont en général suffisant vis-à-vis des fréquences usuelles des perturbations).

Le calcul de la ligne d'arbre est alors un calcul itératif pas à pas (dans le temps) effectué dans cette base modale. 


\section{Paliers avec défauts}

\subsection{Défaut}

Le défaut de forme choisi [11] se présente sous la forme d'une ondulation de la surface de l'arbre résultant d'un défaut d'usinage (serrage dans un mandrin, déformation dues aux vibrations, faux rond de broche). La figure 1 présente le profil (très agrandi) d'un tel arbre et ceci pour deux types de périodes d'ondulation : $2 \pi / 3$ et $2 \pi / 5$. L'amplitude du défaut sera de l'ordre de $10^{-4}$ fois le rayon. Pour un arbre de $40 \mathrm{~mm}$ de rayon ceci représente une ondulation de 4 micromètres.

En lubrification hydrodynamique, les épaisseurs de film sont généralement très faibles, ainsi ce type de défaut peut conduire à des modifications du champ de pression importantes.

En effet les défauts situés sur l'arbre entraînent, même pour un arbre fonctionnant en régime de stabilité sous charge fixe, une apparition d'orbites de fortes amplitudes et dans certains cas des phénomènes de résonances importants, non prévisibles par une étude classique de ligne d'arbre.

Des défauts situés sur l'alésage ont aussi une influence sur le champ de pression, mais cette influence est dynamiquement beaucoup moins préoccupante car elle n'engendre généralement que des perturbations d'ordre statique.

\subsection{Calcul des actions du film sur l'arbre}

La figure 2 présente une section droite d'un palier, où $\mathrm{R}_{\mathrm{a}}$ et $\mathrm{R}_{\mathrm{c}}$ sont les rayons de l'arbre et $\mathrm{du}$ coussinet non déformé. Le repère $(\mathrm{Oc}, X, Y, Z)$ est un repère fixe lié au centre du coussinet Oc. L'arbre est animé d'un mouvement de rotation de vitesse angulaire $\omega_{\mathrm{a}}$. La position du centre de l'arbre peut être décrite par deux systèmes de coordonnées, des coordonnées cartésiennes $(x, y)$ et des coordonnées polaires $(\mathrm{e}, \psi)$ où e est la distance (Oa, Oc) et $\psi$ l'angle entre l'axe $\vec{x}$ et la ligne des centres.

Les défauts d'usinage sur l'arbre sont alors décrits par une composante radiale $\delta_{\text {ra }}$. Le jeu dans le palier restant toujours très petit vis-à-vis du rayon du palier, nous pouvons développer le coussinet et l'arbre et assimiler la surface du coussinet à un plan. Dans ce repère, l'équation des films minces s'écrit alors :

$$
\begin{aligned}
& \frac{\partial}{\partial x}\left[\frac{H^{3}}{\mu} \frac{\partial p}{\partial x}\right]+\frac{\partial}{\partial z}\left[\frac{H^{3}}{\mu} \frac{\partial p}{\partial z}\right]= \\
& 6 \frac{\partial}{\partial x}\left[U_{2} H\right]-12 U_{2} \frac{\partial H}{\partial x}+12 V_{2}
\end{aligned}
$$

Un point courant appartenant à la surface de l'arbre a pour vitesse :

$\mathrm{U}_{2}=\omega_{\mathrm{a}} \mathrm{R}_{\mathrm{a}}$ et $\mathrm{V}_{2}=\dot{\mathrm{e}} \cos (\theta)+\mathrm{e} \dot{\psi} \sin (\theta)+\dot{\delta}_{r a}+\mathrm{R}_{\mathrm{a}} \omega_{\mathrm{a}} \frac{\partial \mathrm{h}}{\partial \mathrm{x}}$

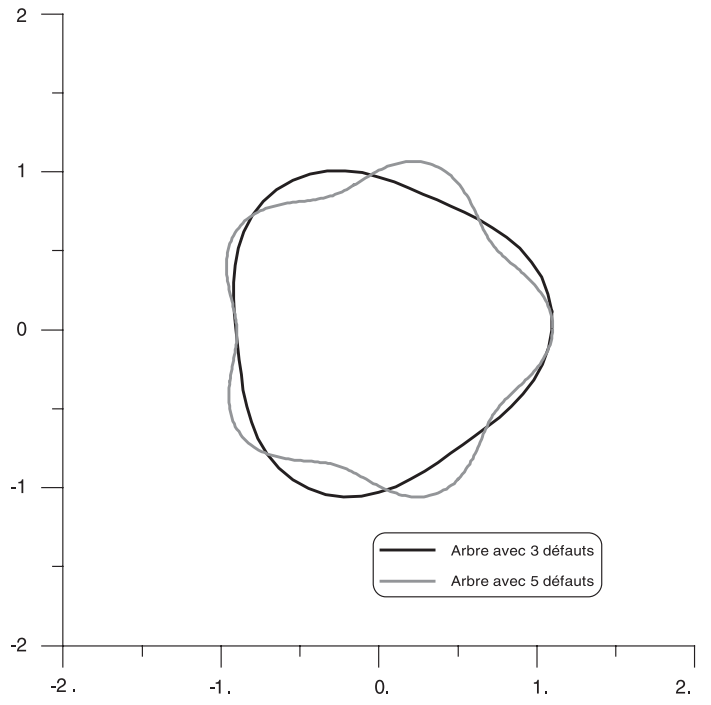

Fig. 1. Profils agrandis d'un arbre avec défauts dans le palier (adimensionnés par le jeu radial).

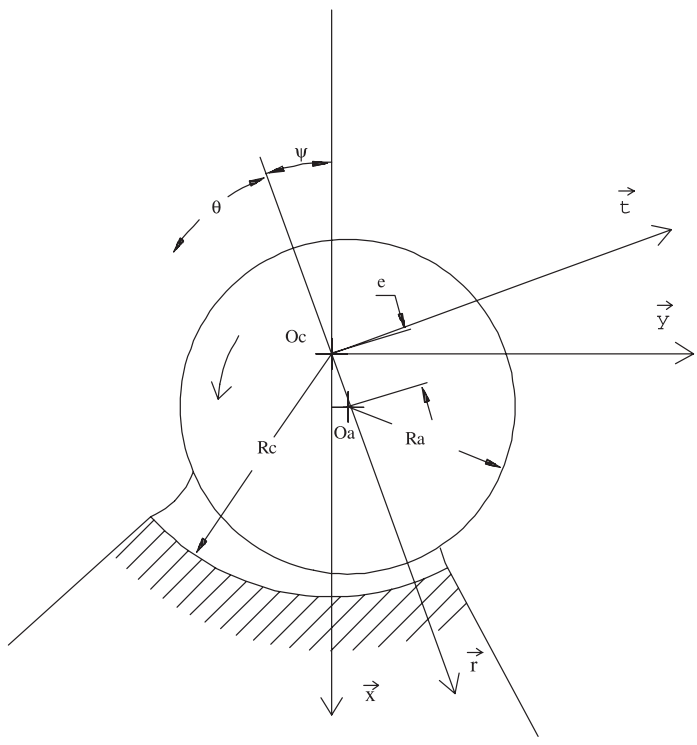

Fig. 2. Schématisation du palier.

Ceci permet d'écrire l'équation des films minces visqueux $[12,13]$ :

$$
\begin{aligned}
\frac{\partial}{\partial x}\left[\frac{H^{3}}{\mu} \frac{\partial P}{\partial x}\right]+\frac{\partial}{\partial z}\left[\frac{H^{3}}{\mu} \frac{\partial P}{\partial z}\right] & =6 \omega_{a} \frac{\partial h}{\partial \theta}-6 \omega_{a} \frac{\partial \delta_{r a}}{\partial \theta} \\
+ & 12\left(\dot{\mathrm{e}} \cos (\theta)+\mathrm{e} \dot{\psi} \sin (\theta)+\dot{\delta}_{r a}\right)
\end{aligned}
$$

Cette équation peut être résolue par la méthode des différences finies avec les conditions aux limites de Reynolds. Cependant l'analyse non linéaire de l'ensemble de l'arbre conduit à des temps de calcul très importants c'est pourquoi nous avons choisi de ne traiter que le cas du palier court. Cette hypothèse permet de simplifier l'équation précédente en supposant que le gradient de pression circonférentielle reste petit devant le gradient de pression axial. Ceci est vrai lorsque la longueur du palier reste 


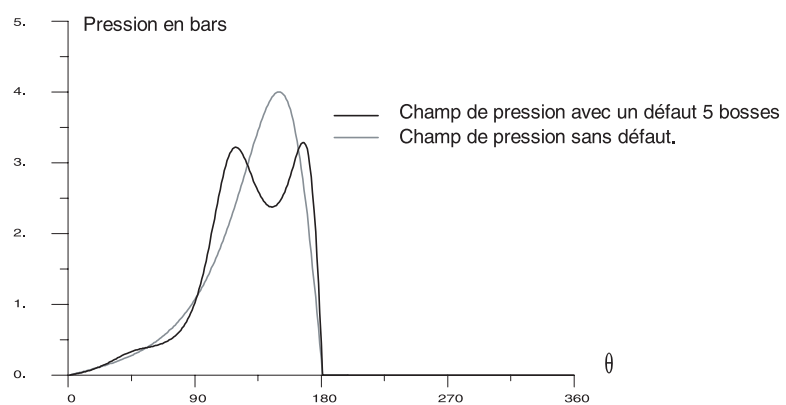

Fig. 3. Champ de pression développé, pour un défaut « 5 bosses ».

petite vis-à-vis des longueurs d'onde du défaut. Compte tenu des dimensions, cette étude est effectuée dans le cas limite de cette hypothèse.

En négligeant le gradient de pression circonférentielle et avec des défauts constants dans le temps $\left(\dot{\delta}_{r a}=0\right)$, l'équation des films minces peut être intégrée et le champ de pression s'écrit :

$$
\begin{aligned}
p(\theta, z)=\frac{6 \mu}{\left(h+\delta_{r a}\right)^{3}}\left[\mathrm{e}\left(\dot{\psi}-\frac{\omega_{a}}{2}\right) \sin \theta\right. & \\
& \left.+\dot{\mathrm{e}} \cos \theta-\frac{\omega_{a}}{2} \frac{\partial \delta_{r a}}{\delta \theta}\right]\left(z^{2}-\frac{L^{2}}{4}\right)
\end{aligned}
$$

Les conditions aux limites sont les suivantes :

- pression d'alimentation à pression atmosphérique aux deux extrémités du palier ;

- condition de Gumbel pour décrire la zone active du film (c'est-à-dire que seule la partie positive du champ de pression est conservée).

Les composantes de la charge suivant les axes $x$ et $y$ sont obtenues par intégration du champ de pression en projection sur ces deux directions.

La figure 3 présente la forme des champs de pression obtenus dans le cas d'un défaut de cinq bosses sur l'arbre et d'un arbre parfait. Nous pouvons remarquer que l'influence du défaut est très sensible : trois pics de pression apparaissent et la pression maximale est sensiblement diminuée par rapport à celle obtenue avec un arbre sans défaut.

Le pic de pression statique (courbe en tirets) est obtenu par l'effet coin d'huile; le champ de pression est fixe par rapport au stator. Les pics existant sur la courbe en trait plein sont obtenus par superposition de l'effet coin d'huile et d'un effet dû à la rotation de l'arbre avec défaut. Cet effet crée un champ de pression tournant avec l'arbre et qui génère un effet dynamique.

\subsection{Résultats}

L'arbre étudié est flexible, monté sur deux paliers. Une étude dynamique non linéaire avec des paliers sans défaut fait apparaître deux premières fréquences de résonance dans le domaine d'étude : la première à $2990 \mathrm{tr} \cdot \mathrm{min}^{-1}$ et la deuxième à $5450 \mathrm{tr} \cdot \mathrm{min}^{-1}$.

\subsection{1 Étude avec faible balourd}

Des montées en vitesses de $1000 \mathrm{tr} \cdot \mathrm{min}^{-1}$ à $6000 \mathrm{tr} \cdot \mathrm{min}^{-1}$ ont ensuite été effectuées avec des paliers comportant deux défauts. L'arbre est soumis à un faible balourd correspondant à des amplitudes de vibrations, dues au balourd, très petites : les fréquences critiques (2990 et $5450 \mathrm{tr} \cdot \mathrm{min}^{-1}$ ) sont alors peu excitées.

La figure 4 présente les amplitudes dans les directions $x$ et $y$. La faible valeur de balourd permet de mettre en évidence l'influence des défauts car l'influence du balourd est alors beaucoup plus faible voir inexistante. Des pics de résonances sont créés à des fréquences égales aux fréquences critiques divisées par le nombre de défauts $\left(1570 \mathrm{tr} \cdot \mathrm{min}^{-1} \approx 2990 / 2 \mathrm{tr} \cdot \mathrm{min}^{-1}\right.$ et $2890 \mathrm{tr} \cdot \mathrm{min}^{-1} \approx$ $\left.5450 / 2 \mathrm{tr} \cdot \mathrm{min}^{-1}\right)$. En effet lorsque l'arbre tourne à une vitesse $\mathrm{N}$ les deux défauts engendrent une force de perturbation dont la fréquence est égale à deux fois N. Une vitesse de l'arbre à $1570 \mathrm{tr} \cdot \mathrm{min}^{-1}$ vient exciter la première vitesse critique de flexion d'où apparition d'un pic d'amplitude.

\subsection{2 Étude avec fort balourd}

La figure 5 présente les courbes obtenues avec un balourd important et un défaut à deux pics. Ceci permet de retrouver les fréquences excitées par les défauts et les fréquences excitées par le balourd (synchrones). Les pics des résonances dues au balourd et aux défauts s'additionnent. Il est intéressant de remarquer la superposition de la fréquence de résonance à $2990 \mathrm{tr} \cdot \mathrm{min}^{-1}$ (balourd) avec celle à $2890 \mathrm{tr} \cdot \mathrm{min}^{-1}$ (défaut deux pics : $5450 \mathrm{tr} \cdot \mathrm{min}^{-1}$ divisé par deux).

\subsection{Bilan}

La prise en compte des défauts sur l'arbre s'avère très importante. En effet nous avons montré que ces défauts faisaient apparaître des pics d'amplitude imprévisibles par une analyse linéaire. L'analyse non linéaire de la ligne d'arbre est ici indispensable. L'hypothèse des petits déplacements dans le palier n'est plus justifiée, les trajectoires présentent des formes inattendues, elles ne sont pas de formes elliptiques et les fréquences de précession peuvent être double ou triple de la vitesse de rotation. Ainsi des défauts du palier de faible amplitude peuvent conduire à l'apparition de trajectoires ayant de très grandes amplitudes même en l'absence de balourd.

\section{Effet des butées}

\subsection{Butées avec défauts}

Les butées hydrodynamiques ont pour rôle de reprendre l'ensemble des charges axiales pouvant exister sur la ligne d'arbre. Elles jouent un rôle prépondérant sur le comportement en vibrations axiales du rotor mais il existe 


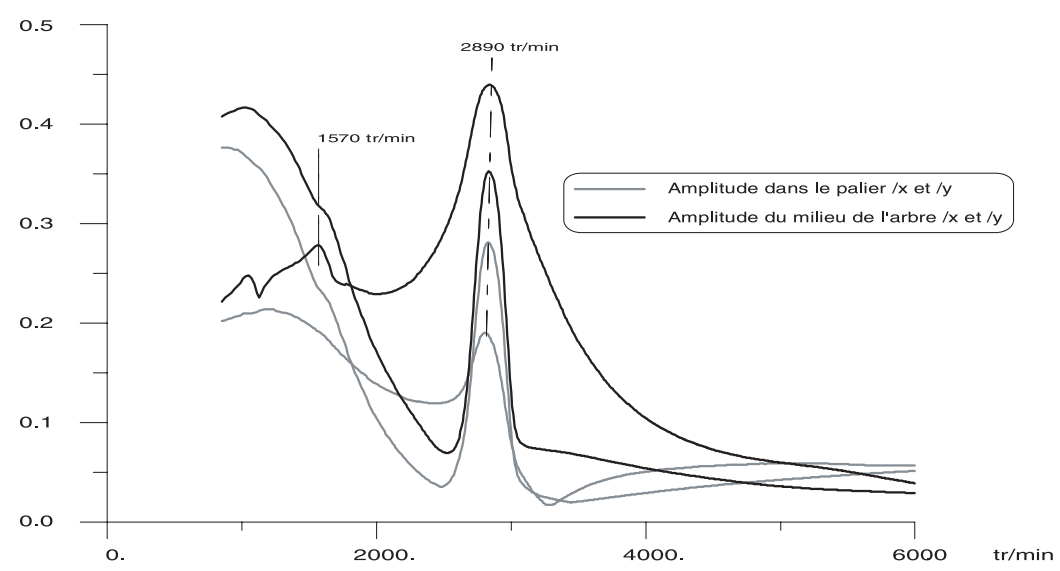

Fig. 4. Amplitudes de vibrations adimensionnées de l'arbre en fonction de $\mathrm{N}\left(\mathrm{tr} \cdot \mathrm{min}^{-1}\right.$ ) (palier 2 défauts, balourd faible).

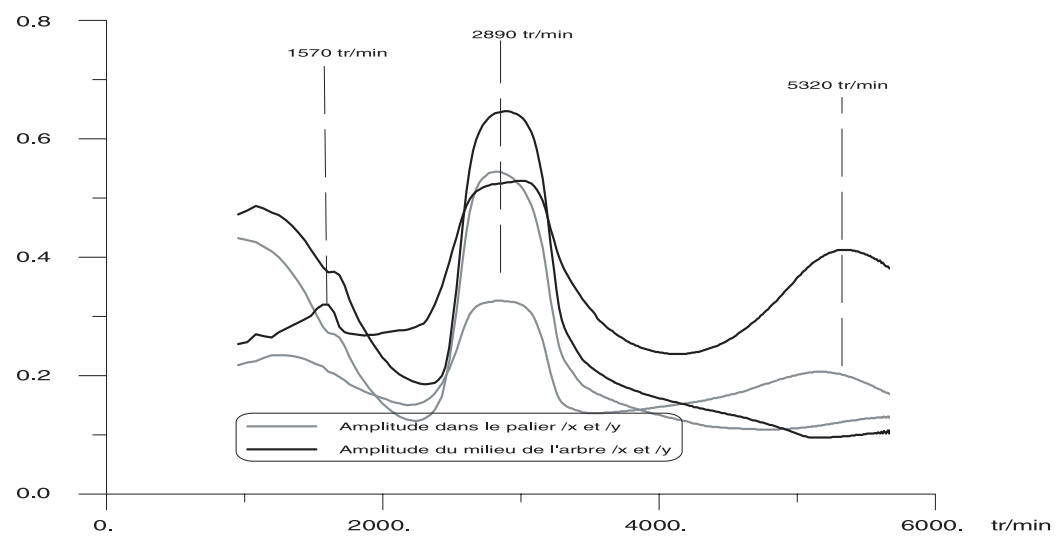

Fig. 5. Amplitudes de vibrations de l'arbre en fonction de $\mathrm{N}\left(\mathrm{tr} \cdot \mathrm{min}^{-1}\right)$ (palier 2 défauts, balourd important).

peu d'études sur ce couplage dynamique [14-20], en particulier en ce qui concerne les non linéarités. Cependant il a été montré [21] que leur rôle sur le comportement des vibrations de flexion pouvait être non négligeable. En effet une simple étude dynamique linéaire permet de mettre en évidence le supplément de raideur en flexion introduit par ces éléments : la flexion de l'arbre se traduisant par un mésalignement de la butée. Ce mésalignement introduit un moment de rappel qui tend à recentrer le rotor. Par ailleurs, un défaut de position (par exemple un défaut de perpendicularité du rotor de butée par rapport à l'axe de rotation de l'arbre) peut entraîner l'apparition de fréquences d'excitation non prévisibles par la théorie linéaire [21-23] et il peut exister des phénomènes de couplage dus à la butée entre les vibrations axiales et radiales. Ce paragraphe présentera les résultats obtenus avec une butée présentant des défauts de forme correspondant à des ondulations de surface du grain mobile. Ces défauts pouvant être dus à un usinage de mauvaise qualité (vibration de machine, relaxation de matériaux...).

La butée est constituée d'un stator formé de quatre patins inclinés de $80^{\circ}$ d'amplitude (cf. Fig. 6) et d'un rotor lisse présentant le défaut d'ondulation (défaut à deux vagues et à trois vagues).

L'équation de Reynolds permet alors de décrire le comportement du film d'huile. L'intégration, par différences finies, de cette équation permet d'obtenir le

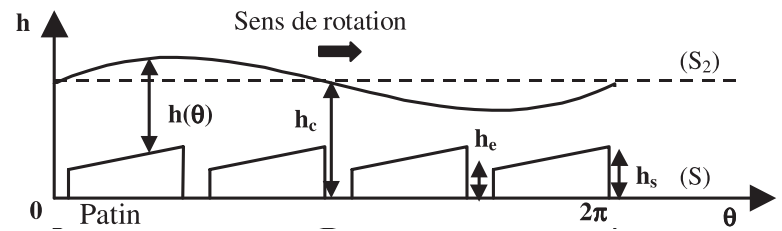

Fig. 6. Vue développée du palier avec un défaut comportant deux vagues.

champ de pression sur le rotor et par conséquent d'obtenir les actions mécaniques du film sur l'arbre. Ces calculs maintenant classiques ont été présentés par ailleurs [24].

\subsection{Résultats}

La figure 7 montre schématiquement l'arbre supporté par deux paliers et par une butée axiale chargée de reprendre l'effort axial $\mathrm{F}_{\mathrm{a}}$.

Le diamètre intérieur de la butée est de $0,04 \mathrm{~m}$ et son diamètre extérieur de $0,08 \mathrm{~m}$. L'épaisseur du film (qui dépend de la charge axiale) est comprise entre 100 et $160 \mu \mathrm{m}$. L'arbre a une longueur de $0,8 \mathrm{~m}$ pour un diamètre de $0,06 \mathrm{~m}$. 


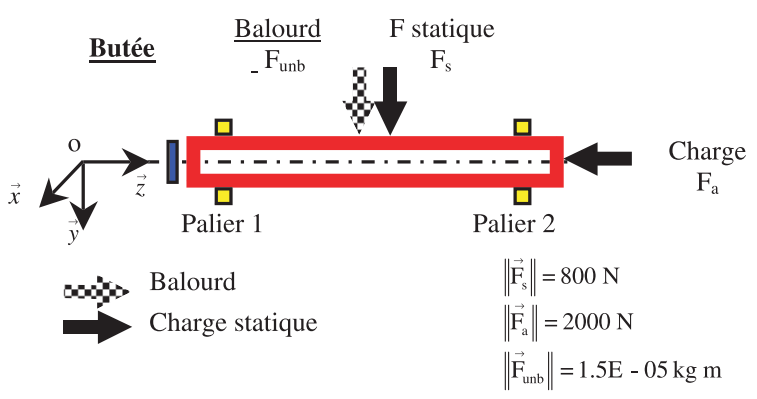

Fig. 7. Représentation de l'arbre et des chargements.

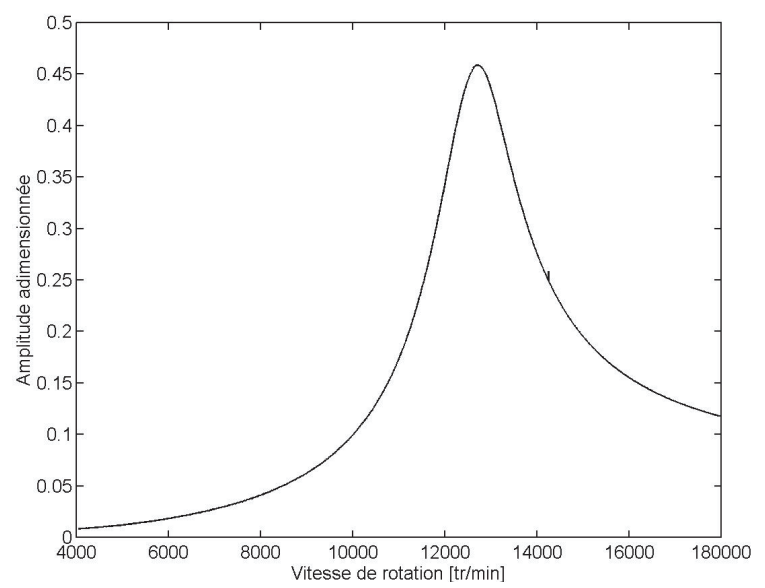

Fig. 8. Amplitude adimensionnée du mouvement milieu du rotor lors d'une montée en vitesse (butée sans défaut - avec balourd).

Cet arbre est soumis à des charges statiques radiales et axiales auxquelles peut se superposer une charge dynamique radiale de type balourd.

Différentes montées en vitesses ont été effectuées afin de permettre la caractérisation du comportement de l'ensemble.

Le premier calcul va permettre d'identifier le comportement dynamique de la ligne d'arbre. Il s'agit d'une montée en vitesse (2000 à $\left.18000 \mathrm{tr} \cdot \mathrm{min}^{-1}\right)$ avec un balourd mais sans aucun autre défaut. Une fréquence de résonance (cf. Fig. 8) existe à $12700 \mathrm{tr} \cdot \mathrm{min}^{-1}$ avec un pic d'amplitude radiale (pour le centre du rotor) de l'ordre de 0,45 fois le jeu radial des paliers $(30 \mu \mathrm{m})$.

La deuxième série de calculs a été effectuée sans aucune charge dynamique extérieure (pas de balourd) mais avec une butée présentant des défauts de forme. Deux cas sont traités : un défaut avec deux vagues et un défaut à trois vagues. Ces défauts ont une amplitude de $10 \mu \mathrm{m}$.

La figure 9 présente l'amplitude de la trajectoire du milieu du rotor lors d'une montée en vitesse; un pic de résonance est présent sur chacune des courbes. Il se développe vers $6400 \mathrm{tr} \cdot \mathrm{min}^{-1}$ dans le cas d'une butée deux défauts et vers $4300 \mathrm{tr} \cdot \mathrm{min}^{-1}$ pour la butée trois défauts. Il s'agit dans chaque cas de l'apparition d'une charge dynamique tournante de fréquence égale à la fréquence de rotation multipliée par le nombre de défaut. C'est pourquoi l'arbre entre en résonance à $12700 / 2 \mathrm{tr} \cdot \mathrm{min}^{-1}$ et $12700 / 4 \mathrm{tr} \cdot \mathrm{min}^{-1}$. Les amplitudes

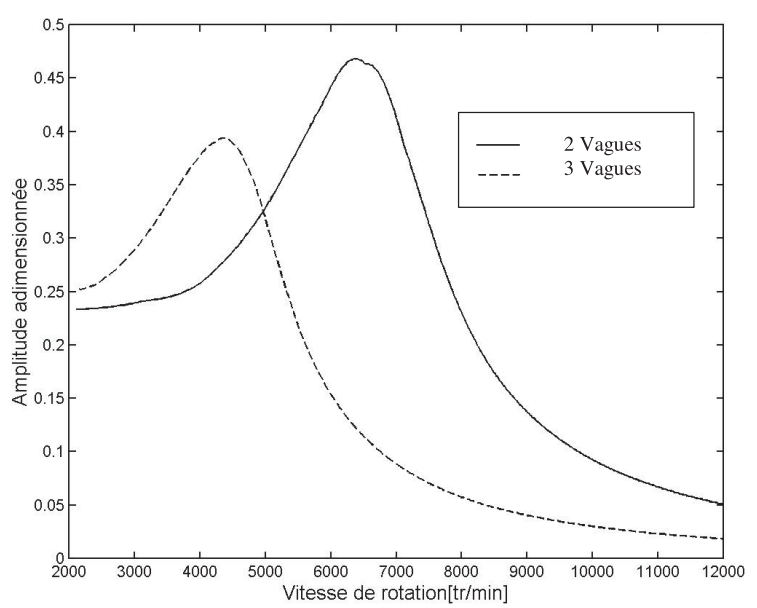

Fig. 9. Amplitude adimensionnée du mouvement milieu du rotor lors d'une montée en vitesse (butée avec défauts 2 vagues et 3 vagues en absence de balourd).

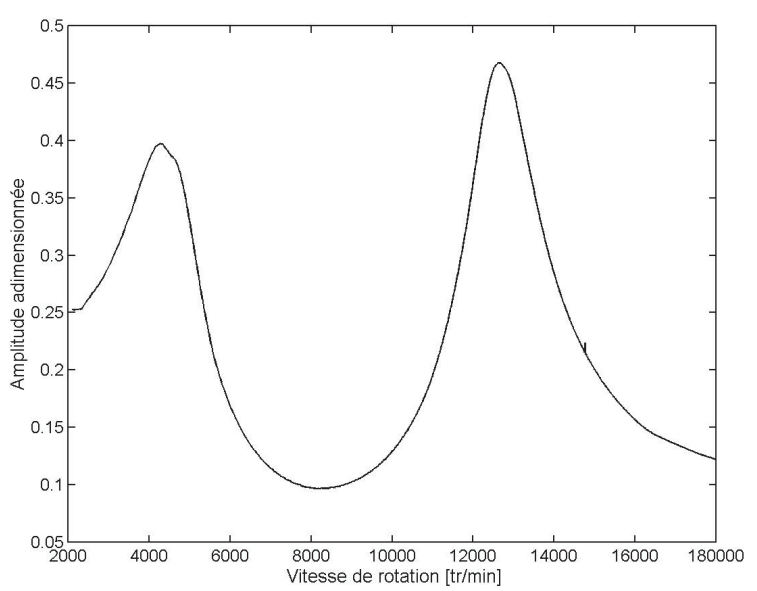

Fig. 10. Amplitude adimensionnée du mouvement milieu du rotor lors d'une montée en vitesse. (Butée avec défauts 3 vagues en présence de balourd.)

sont ici assez importantes et du même ordre de grandeur que celles obtenues avec le balourd.

Le dernier cas étudié ici concerne le comportement du rotor soumis à un balourd et supporté par une butée avec trois défauts. La figure 10 montre l'existence de deux pics. Ces deux pics sont dus au premier mode de vibration excité d'une part par la butée défectueuse (premier pic à $4300 \mathrm{tr} \cdot \mathrm{min}^{-1}$ ) et d'autre part par le balourd (pic à $\left.12700 \mathrm{tr} \cdot \mathrm{min}^{-1}\right)$.

\subsection{Bilan}

L'influence d'un défaut de forme du grain mobile d'une butée hydrodynamique peut conduire à l'existence de charges dynamiques d'excitation non prévisibles par des études classiques. Les amplitudes à la résonance peuvent être importantes. Le phénomène présente certains caractères de linéarité. Ainsi des défauts deux fois plus petits conduisent à des amplitudes deux fois plus petites. De même les fréquences dépendent directement du nombre de 
défauts. Cependant, seule une analyse non linéaire pas à pas de la butée permet d'obtenir des résultats réalistes.

\section{Conclusion générale}

Les résultats présentés dans cet article mettent en évidence l'intérêt des études couplées faisant intervenir le calcul non linéaire des supports de ligne d'arbre et la prise en compte des flexibilités du rotor. En effet il est clair que les phénomènes à l'origine du comportement des lignes d'arbres peuvent difficilement être isolés. Le couplage entre le comportement dynamique du rotor (en particulier la prise en compte de sa flexibilité) et le comportement non linéaire des supports fluides (paliers, butées) permet d'expliquer des phénomènes que les analyses classiques ne peuvent pas mettre en évidence. Une modélisation linéaire de palier (modélisé par des matrices de raideurs et d'amortissements), par exemple, ne pourra jamais traduire les effets dus à des défauts de forme. Bien sûr les études classiques, pour laquelle les composants (aussi bien supports que ligne d'arbre) sont utilisés dans des conditions « usuelles », restent une approche intéressante qui permet d'appréhender le dimensionnement statique et dynamique. Mais il faut rester prudent et toujours bien analyser les résultats au regard des hypothèses formulées.

L'intérêt des études menées ici est qu'elles nous ont permis de mieux comprendre la physique des phénomènes mis en jeu. La présence de défauts, par exemple, engendre des pics de pression dynamiques qui viennent exciter des modes de résonance de la structure. Il est ainsi possible d'expliquer l'apparition de pics d'amplitude qui n'étaient absolument pas prévisibles autrement.

\section{Références}

[1] J. Lacroix, Comportement dynamique d'un rotor au passage des vitesses critiques, Thèse, INSA de Lyon, 1988

[2] M. Lalanne, G. Ferraris, Rotordynamics Prediction in Engineering, Wiley, Chichester, 1990

[3] J.W. Lund, K.K. Thomsen, A calculation method and data for the dynamic coefficients of oil lubricated journal bearing, ASME, Topic in fluid film bearing and rotor bearing system design and optimisation, 1978, pp. 1-18

[4] N. Abdul-Wahed, Comportement dynamique des paliers fluides. Étude linéaire et non linéaire, Thèse de doctorat d'état, Université Claude Bernard, Lyon, 1982

[5] B. Sternlicht, Elastic and damping properties of oil film journal bearing for application to unbalance vibration of calculation, ASME, J. Appli. Mech. 80 (1958) 141

[6] O. Bonneau, Comportement statique et dynamique de ligne d'arbre montée sur paliers fluides : influence des caractéristiques des paliers, Thèse, université de Poitiers, 1989

[7] O. Bonneau, A. Kassai, J. Frêne, J. Der Hagopian, Dynamical behavior of an elastic rotor with a squeeze film damper, Proceeding EUROTRIB, Helsinki, Finland, juin 1989, Vol. 4, pp. 145-149

[8] A. Kassai, Contribution à l'étude dynamique des rotors amortis, Thèse, INSA de Lyon, 1989
[9] O. Bonneau, J. Frêne, Numerical study of a flexible rotor mounted in an active squeeze film damper, Fourth International Conference on Rotor Dynamics, IFToMM, 1994, Chicago, pp. 327-331

[10] B. Pecheux, O. Bonneau, J. Frêne, Investigation about Electro-Rheological Squeeze Film Damper Applied to Active Control of Rotor Dynamic, International Journal of Rotating Machinery 3 (1997) 53-60

[11] O. Bonneau, J. Frêne, Influence of Bearing Defects on Dynamical Behavior of an Elastic Shaft, International Journal of Rotating Machinery 2 (1996) 281-287

[12] D. Berthe, B. Fantino, J. Frêne, M. Godet, Influence of the shape defects and surface roughness on the hydrodynamics of lubricated systems, Journal Mechanical Engineering Science. Institution of Mechanical Engineers 16 (1974) 156-159

[13] Berthe D., Godet M., Équation de l'écoulement laminaire entre deux parois rapprochées en mouvement relatif, C.R. Académie des Sciences, Paris, 1971, t. 272, série A, pp. 1010-1013

[14] P.L. Jiang, L. Yu, Effect of a hydrodynamic thrust bearing on the statics and dynamics of a rotor-bearing system, Mechanics Research Communications 25 (1998) 219-224

[15] N. Mittwollen, T. Hegel, J. Glienicke, Effect of hydrodynamic thrust bearings on lateral shaft vibrations, Journal of Tribology 113 (1991) 881-818

[16] T. Someya, M. Fukuda, Analysis and experimental verification of dynamic characteristics of oil film thrust bearings 15 (1972) 1004-1015

[17] M.C. Jeng, A.S. Szeri, A thermohydrodynamic solution of pivoted thrust pads, Journal of Tribology 108 (1986) 195-218

[18] Q. Zhu, Y.B. Xie, L. Yu, Axial transient forces of thrust bearing rotor system in a turbo-expander, Proceedings of International Conference on Hydrodynamic BearingRotor System Dynamics, China, 1990

[19] P.L. Jiang, L. Yu, Effect of a hydrodynamic thrust bearing on the statics and dynamics of a rotor-bearing system, Mechanics Research Communications 25 (1998) 219-224

[20] N. Mittwollen, T. Hegel, J. Glienicke, Effect of hydrodynamic thrust bearings on lateral shaft vibrations Journal of Tribology 113 (1991) 881-818

[21] S. Berger, Étude du comportement dynamique non linéaire d'un rotor monté sur éléments fluides : caractérisation des étanchéités - Influence de la butée, Thèse, Université de Poitiers, 1999

[22] S. Berger, O. Bonneau, J. Frêne, Influence of axial thrust bearing defects on the dynamic behavior of an elastic shaft, Proceedings of the 5th International Tribology Conference Austrib'98, Brisbane, 6-9 December 1998, pp. 389-394

[23] S. Berger, O. Bonneau, J. Frêne, Influence of Axial Thrust Bearing on the Dynamic Behavior of an Elastic Shaft: Coupling between the Axial Dynamic Behavior and the Bending Vibrations of a Flexible Shaft, Journal of Vibration and Acoustics 123 (2001) 145-149

[24] J. Frêne, D. Nicolas, B. Degeurce, M. Godet, D. Berthe, 1990 Lubrification hydrodynamique, paliers et butées, Edn. Eyrolles, collection direction études et recherche d'EDF, 1990 